\title{
Enriching accounts of computer-supported collaboration by using video data
}

\author{
Canan Tosunoglu Blake and Eileen Scanlon \\ The Open University \\ email:c.tosunoglu@open.ac.uk; e.scanlon@open.ac.uk
}

This paper will discuss the approach to the evaluation of computer-supported collaborative learning developed in our group over the past ten years. This approach depends on the collection of video data to allow the analysis of key features of problemsolving behaviour within groups of students working on collaborative learning tasks. Our theoretical framework derives from two sources - the CIAO! framework for evaluating examples of CAL and an analysis of appropriate methods of evaluating computersupported collaboration. Our work in this area has been supported by developing the data capture facilities for the CALRG (Computers and Learning Research Group) at the Open University: We will draw on a number of studies to illustrate this approach and will present a brief case study from work done on a computer-supported learning environment for statistics where we use video records of video-mediated collaboration. This case study gives an example of the rich data that can be collected using video recording and analysed to increase understanding of computer-supported collaboration.

\section{Introduction}

Members of CALRG (Computers and Learning Research Group) at the Open University have been carrying out experiments investigating the usefulness of technology-mediated collaborative problem-solving as part of an ongoing research programme. Our approach to analysing computers and learning in the group has been based on a strong empirical tradition, as many of our original studies were with students of the Open University. As a result our work has been closely associated with a desire to improve the experience of learning for our students.

We developed the context, interaction, attitudes and outcomes (CIAO!) framework to support the evaluation of CAL developments, mainly for the Open University courses (Jones, Scanlon, Tosunoglu, Morris, Ross, Butcher and Greenberg, 1999). In this 
framework, information from a variety of sources and both qualitative and quantitative techniques are used. Evaluation teams include CAL designers, course developers and educational technologists and have access to the stated aims, rationales and knowledge about the context of use of the CAL programmes under investigation. This approach enabled us to evaluate the effectiveness and quality of CAL whilst simultaneously investigating the educational situation as a whole and focusing on the learners. The overall emphasis is on educational issues and in pursuing these issues it is necessary to observe students interacting with the educational innovation. Issroff (1995) presents an approach to the evaluation of computer-supported collaborative learning situations by stressing that there needs to be a wider consideration of the potential benefits of such interventions. She stresses the importance of affective measures and recording interactions.

Analysing students' interactions with educational software gives us a window through which to examine learning processes. This can provide information on the effect of particular elements of instruction and why they work. Examining the interactions that students have with computers and with each other requires observational data, preferably supported by video data.

This emphasis on the process requires a detailed capture of the learning setting and is best realized by using a relatively objective observation tool such as video (using video is not entirely objective because choices made in the set-up, such as positioning of the video camera and microphone, have an impact). Video data also allows the analysis to be carried out collaboratively by more than one researcher and different aspects of the investigation can be addressed by using qualitative and quantitative techniques. Video and film records are considered to be essential for the qualitative study of human behaviour in areas such as analysis of motor activities and non-verbal communication. It is also possible to use video to collect quantitative data, such as recording the amount of time spent by participants on different aspects of a task (for example, planning, experimenting, discussion). Video is particularly significant in many of our studies which involve analysing users' collaborative problem-solving, in particular how they verbalize their understanding to each other and how they learn together.

Video is an increasingly popular tool for educational researchers. In the past, video-taped data has been used mainly in small, qualitative studies. With the new developments in storage capabilities of computers and processor speeds, video is becoming a practical tool to study teaching and learning settings in a much more comprehensive way. It is now possible to store many hundred hours of video-taped data in computers and analyse them relatively easily using sophisticated analysis software (Jacobs, Kawanaka and Stigler, 1999). The software allows users to view video, create a transcript and link places in the transcript to the corresponding frames in the video. It is also possible to identify and organize the significant clips of video using the tools provided. Jacobs et al. (1999) describe an approach which has been used for large-scale cross-cultural study of mathematics classrooms. Our use of video has been more localized and consists both of video collected in a laboratory and a naturalistic setting. The next section explains the equipment used in our experiments. 


\section{The data capture suite}

A data capture suite has been developed to capture video records of interaction, which can combine video recerds of each user with a synchronous record of their computer screen. These can be displayed in a four-way matrix (see Figure 1). For pairs of learners this meant capturing each user's screen and their videoconferencing record. For larger groups a single shared screen can be recorded with each individual user's video. In some experiments, two users are in separate rooms with a workstation each, and communicate through a variety of modes. The modes of communication include either a high fidelity, hands-free audio link, with videoconferencing or a specialized videoconferencing device. At the same time, the data from the computer screen is converted into video format by using a scan converter. This set-up allowed us the simultaneous viewing of participants' verbal and non-verbal communication and their interaction with the computer simulation, therefore facilitating the detailed analysis of the session.

Video cameras were used to record task performance and interaction with the computer. One camera was used to record task performance. One camera captures the information displayed on screen. This data collection set-up was inspired by the development of the media space at Xerox PARC in the mid-1980s (see Bly, Harrison and Irwin, 1993), and then developed further in the Rank Xerox Research Centre, Cambridge, where our first experiences with this technology were developed on joint projects (see, for example, Smith et al., 1989).

We analyse our records by looking at a verbal protocol and by relating utterances made by participants to both events in working with the computer and any non-verbal communication.

Figure 1: The four-way matrix comprising four different video screens with two subjects, the

observer and the computer simulation screen

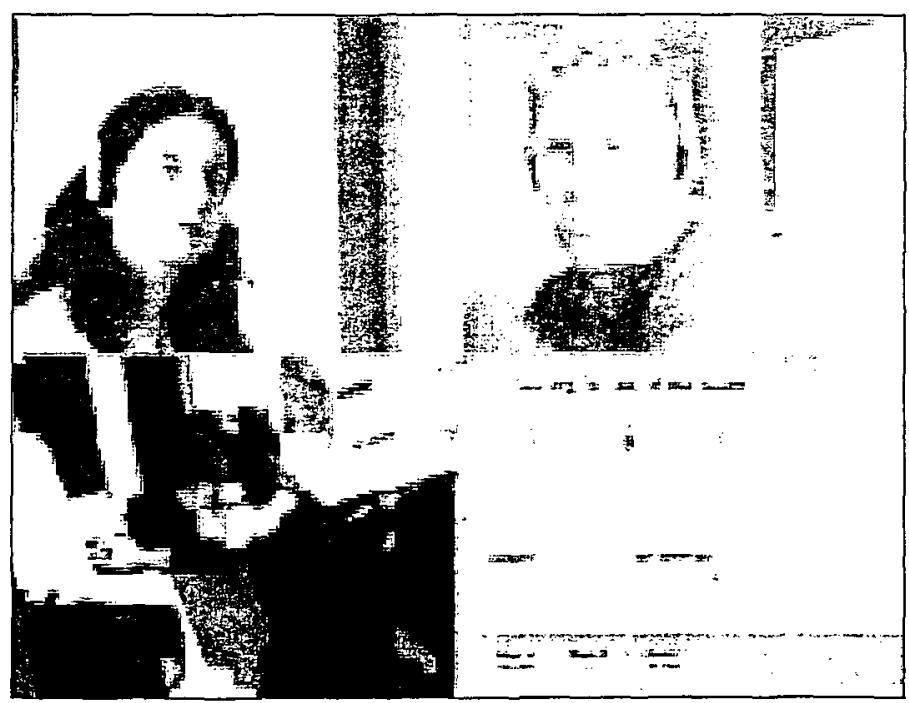

\section{Previous studies}

We used the data capture suite to study several different computers and learning environments. One example was a physics problem-solving tool, Physica, developed for 
students studying the Open University's introductory physics course (Bolton, 2001). Physica includes customized tools designed to help students to solve problems and to perform the routine tasks in algebra and calculus that are needed for the course. During its development Physica was used by a few pairs of student volunteers to help designers observe the problem-solving behaviour of real students and provide necessary tools and facilities in the program. Students were also interviewed after their session with Physica. This study lead to various improvements of the interface, including the introduction of highlighting as a major mechanism for identifying variables and equations. Using the problem-solving facility of Physica requires manipulating multiple windows and extensive menus to perform actions such as customizing or solving equations. Without video data showing how students used several different components of the Physica environment, it would be very difficult to design an effective interface.

We have also used video to evaluate computer-supported collaborative learning in a more naturalistic setting, this time involving individuals and pairs of secondary school children aged between thirteen and fourteen years old using a chemistry database to fill in a worksheet about the Periodic Table. Empirical investigations by Issroff, Scanlon and Jones (1997) revealed that, as in many other evaluation studies, there was no clear cognitive benefit from working in a pair when learning outcomes were measured by pre- to post-test shifts alone. However, the perceptions and motivations of students in two conditions were different. Students in the paired condition found the experience more positive, enjoyable and motivating. Conclusions from this study focused on the usefulness of considering what time was spent by individuals on different sorts of activity and the importance of records of collaborative working which preserve the timeline of the collaboration. To explore this further, video-tape records of the children were analysed in terms of talk and behaviour. Four categories of talk were used: topic, next, control and other. The behaviours that were used were mouse use, typing, reading, writing, other, researcher present and looking at the Periodic Table. This type of analysis produced summary tables and time-based plots of the talk and behaviour. The analysis was applied to ten pairs of students and five individuals. The analysis found inter-pair, intra-pair and inter-individual differences, and the timelines and summaries from pairs who worked for more than one session showed developments over time during the interactions (for an overview of this, see Issroff, Jones and Scanlon, 1994). Time-based analyses can therefore be very valuable, especially when collaborations which occur over more than one session are investigated. It remains a focus of interest in the group to explore efficient ways of analysing video-tape records.

Among the other settings which have been explored using rich video accounts have been teenagers learning the laws of momentum (Whitelock and Scanlon, 1996), children working on the phases of the moon (Whitelock, del Soldato, Scanlon and Taylor, 1996), adults looking at an astronomy web site, adults learning applied maths (Smith et al., 1989) and healthcare professionals using CoMET (Concept Modelling Environment for Teachers) to investigate the educational potential of a concept-based toolkit (Alpay and Giffen, 1998).

We shall illustrate our methods further by presenting a case study of two adults collaborating together to solve a statistical problem (Scanlon, O'Shea, Smith and Joiner, 2000). 


\section{Case study}

In our most recent phase of studies, we are investigating the problem-solving behaviour of pairs of adults working on a statistical problem. ${ }^{1}$ Users each have a window in which they can see a simulation and manipulate it. The subject of the simulation for the study was 'The Monty Hall Problem' that gets its name from the 1970s American television game show, Let's Make A Deal, hosted by Monty Hall. In the game show you are given the opportunity to select one closed door of three, behind only one of which there is a prize. Once you have made your selection, the game show host will open one of the remaining doors, revealing that it does not contain the prize, then asks you if you would like to switch your selection to the other unopened door, or stay with your original choice.

Our two participants are first introduced to the problem by using a standard script and then asked to fill in a pre-test questionnaire asking them individually what they would do switch or stay. Then the session begins and they are given the chance to use the simulation with the experimenter acting as a 'game show host'. The two are then asked to come to a conclusion together about what is the best strategy. After the session they fill in a post-test questionnaire.

We are interested in how the simulation and the collaborative working situation impacts on the participants' understanding of the problem. To explore this we have run a number of different experiments, altering the setting each time. In each case our focus has been on analysing video records of the computer-supported collaboration in an iterative way. Some preliminary accounts of this work which focus on the aspects of eye contact include Joiner, Scanlon, O'Shea, Smith and Blake (2002) and Scanlon et al. (2000).

\section{The problem-solving experience}

Frances (female) and Colin (male) were our participants. They worked together on this task for 45 minutes, communicating via a video link. Colin had more mathematical experience than Frances. In order to progress through the problem, Frances and Colin were required to:

1. make their initial decisions;

2. assess possible courses of action (i.e. stick with the door they originally chose, switch to the other unopened door, or decide it makes no difference);

3. design an experimental strategy to see which is the best course of action, using the simulation;

4. interact with the game show host and the simulation to find the answer; and

5. explain the answer and agree on a solution.

Both participants initially said they would stick to their original decision, Colin because it was 'psychologically better' and it would be worse if you lose after changing from the correct choice, and Frances because she trusted her instincts. They did not make any plans for an experimental test but just started to try things out. After only three experiments, they repeated their original decision to stick. The experimenter suggested that they might carry out a few more trials since their sample size was quite small. They agreed and decided 
to check out a "changing' strategy. In about ten trials they always won. They were surprised and after a little discussion they both said they had changed their minds about the best strategy; they had decided that it was better to change (see Figure 2 for an extract of their discussions). Colin tried to explain the result in terms of probabilities, and suggested that after the opening of the 'wrong' door by the experimenter the probability of choosing correctly was 50 per cent and greater than the initial probability of $1 / 3$. This is an example of a misconception in assigning probabilities to independent and dependent events. It also shows that the participants were trying to match the findings of their experiment to their expectations.

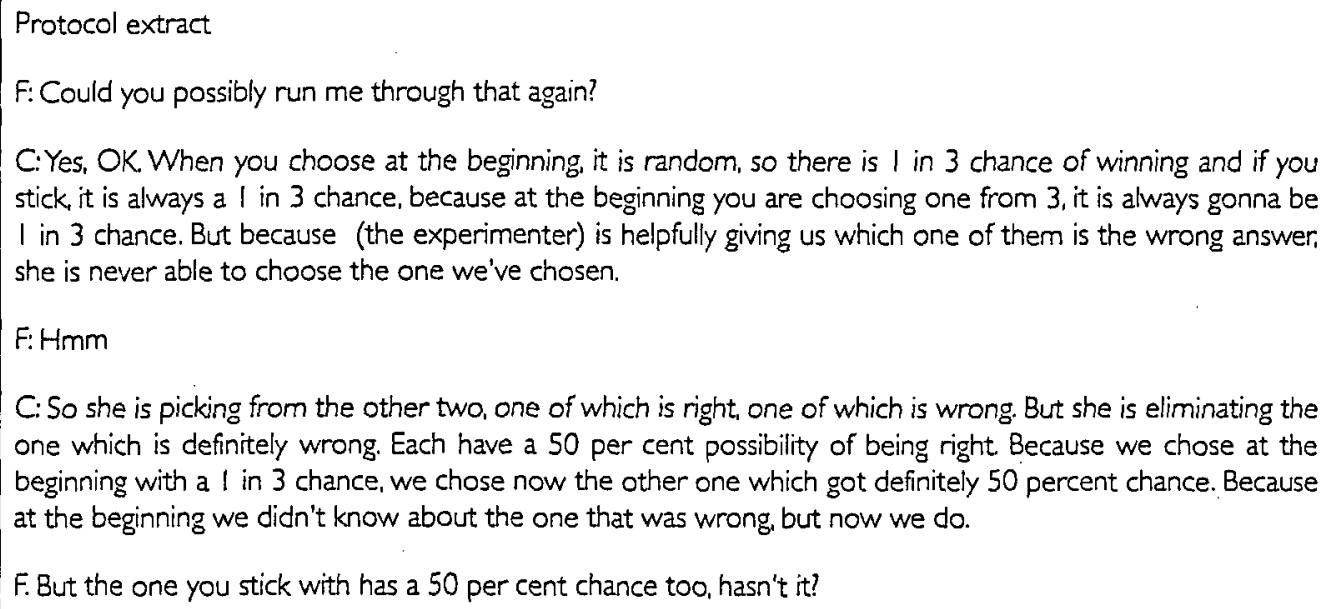

Figure 2: Protocol extract

\section{Use of the video record to understand the problem-solving experience}

Reviewing the video record was very important in allowing us to construct a picture of the collaborative problem-solving experience. On a general level it enabled us to explore how the two participants behaved after the solutions had been agreed upon. For example, Frances first agreed to Colin's explanation but after they stopped, she asked him to explain again, because she could not understand why the probabilities were different for the newly selected door and for the original door. While trying to justify/explain the solution to Frances, Colin went over the probabilities again and realized that the two doors together had a probability of $2 / 3$. Frances was happy with this explanation. Interestingly, Colin and Frances did not make any notes of the trials or share any information regarding their data.

In most cases, after the session ended the participants continued to talk about the task, to explain why they would or would not change, why they agreed, what they were confused about, and they also wanted to know more about the task. These informal discussions after the session were helpful in interpreting the actions during the session. For example, Colin said that his partner's questioning led him to think harder and at the end he realized that the solution was different from what he had initially thought. Frances also admitted that (in the absence of her partner) the explanation was not very clear to her but she believed. him. 
If Colin had been working on his own, perhaps he would not have gone so far in trying to explain the solution to his partner. Seeing her unconvinced, although apparently in agreement, probably made him think harder. Although her verbal protocol suggested at all times that she was agreeing with him, it is clear from examination of the video-tape that she was sending him non-verbal cues which suggested that she was confused or unconvinced. It is also clear from the tape that, on occasions, the use of a particular tone of voice signals other factors, such as the degree of confidence felt by Colin about the assertion he has just made.

Other specific uses of the video record were made including examining the talk time distribution of participants in the session (see Figure 3). The data presented in Figure 3 is an example of the data generated from analysis of a twenty-minute section of activity. It shows how many seconds each participant had talked in the different major subdivisions of activity. To obtain this data we used Transana, ${ }^{2}$ a video analysis and transcription program, and time-coded each piece of speech by participants. Such data is then used to compare the activity levels of each subject in each stage of the collaborative work.

Figure 3: Video analysis of how long participants talked at different stages of the session

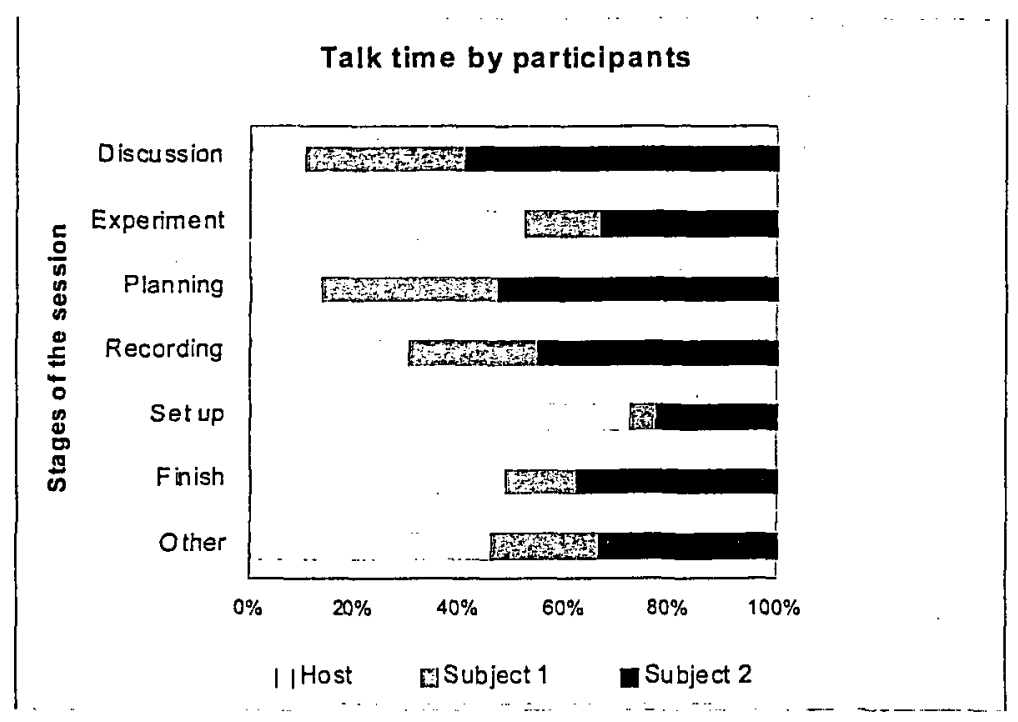

\section{Conclusions from the case study}

From this case study we have drawn conclusions about the impact of the experience on the subjects' problem-solving and about the value of video records in examining this.

This account shows the benefits to us in detailed analysis of interactions in assessing learning events. If we were unable to look at detailed records we would be reduced to giving an account of the impact of this experience based on whether the post-test questionnaire had been adequately completed. Our record shows that learning outcomes are much more complicated than this. Both participants made progress with the task (although occasionally they forgot the progress they had made). In addition, the progress was sometimes due to the effects of explaining their thoughts to one another as much as examining the results of their joint experiments. 
Video data made it possible to observe the subjects' non-verbal gestures. We were able to look at the video for evidence about the degree of certainty with which the participants put forward their suggestions or solutions to each other and what their reactions were to their partner's suggestions. A verbal protocol does not always contain clues to these behaviours. This example is part of a larger database with which we are beginning to explore the value of videoconferencing and eye contact during remote problem-solving.

\section{Main conclusions and future work}

We have reviewed here our approach to the evaluation of computer-supported collaborative learning developed in our group over the past ten years. We outlined the reasons for emphasizing the collection of video data to allow the analysis of key features of problem-solving behaviour within groups of students working on collaborative learning tasks, given an illustration with one example of the type of data we collect and the type of analysis which can be supported.

Within the CALRG group we are continuing to develop our methods of collecting and analysing video data. Current projects include internally focused work on the development of software and Web-based teaching for Open University students and externally funded research projects investigating students' use of remote laboratories (see, for example, DiPaolo and Scanlon et al., forthcoming) in a European consortium and the development of teaching using digital maps in a UK setting (Blake, Jones, Davies and Scanlon, 2003).

\section{Notes}

1. In the experiments we used the Kansas system developed by Randall Smith at Sun Microsystems.

2. http://www.transana.org/

\section{Acknowledgements}

We wish to thank all members of the CALRG who have contributed to this development over the past ten years and of course to our subjects for giving their time.

\section{References}

Alpay, L. and Giffen, R. (1998), 'Investigating a concept-based tool to enhance the process of curriculum development: an example for healthcare education', in B. Cesnik, A.-T. McCray and J.-R. Scherrer (eds), Proceedings of MEDINFO' 98 (9th World Congress on Medical Informatics), Seoul, Korea: IOS Press, 781-4.

Bly, S., Harrison, S. and Irwin, S. (1993), 'Media spaces: bringing people together in a video, audio and computing environment', Communications of the $A C M, 36,28-45$.

Bolton, J. P. R. (2001), 'Physica and problem-solving in physics', in Y. Tazawa (ed.), Symbolic Computation: New Horizons, Proceedings of the Fourth International Mathematica Symposium, Tokyo: Tokyo Denki University Press.

Blake, C. T., Davies, C., Jones, A., Morris, E. and Scanlon, E. (2003), 'Evaluating complex digital resources', $A L T-J, 11,1,4-16$. 
DiPaolo, T., Scanlon, E. with Colwell, C., Cooper, M., Uren, V. and Jelfs, A. (forthcoming), in R. Holliman and E. Scanlon (eds), Mediating Science Learning through ICT, London: Routledge.

Issroff, K., Scanlon, E. and Jones, A. (1997), 'Two empirical studies of computersupported collaborative learning in science: methodological and affective implications', Proceedings of Computer Support for Collaborative Learning 1997, 117-23.

Issroff, K. (1995), 'Investigating computer-supported collaborative learning from an affective perspective', unpublished Ph.D. thesis, Institute of Educational Technology, Open University, Milton Keynes.

Issroff, K., Jones, A. and Scanlon, E. (1994), 'Case studies of children cooperating with computers: a time based analysis', in H. C. Foot, C. J. Howe, A. Anderson, A. K. Tolmie and D. A. Warden (eds), Group and Interactive Learning, Southampton/Boston: Computational Mechanics Publications.

Jacobs, J. K., Kawanaka, T. and Stigler, J. (1999), 'Integrating qualitative and quantitative approaches to the analysis of video data on classroom teaching', International Journal of Educational Research, 31, 717-24.

Joiner, R., Scanlon, E., O'Shea, T., Smith, R., and Blake, C. (2002), 'Evidence from a series of experiments on video mediated collaboration: does eye contact matter?', in G. Stahl (ed.), Proceedings of Computer Support for Collaborative Learning: Foundations for a CSCL Community, Boulder: University of Colorado, 371-8.

Jones, A., Scanlon, E., Tosunoglu, C., Morris, E., Ross, S., Butcher, P. and Greenberg, J. (1999), 'Contexts for evaluating educational software', Interacting with Computers, 11, 499-516.

Scanlon, E., O'Shea, T., Smith, R. B. and Joiner, R. (2000), 'Technological mediation of synchronous collaboration: science and statistics in SharedArk and Kansas', in R. Joiner, K. Littleton, D. Faulkner and D. Miell (eds), Rethinking Collaborative Learning, London: Free Association Books.

Smith, R. B., O’Shea, T., O'Malley, C., Scanlon, E. and Taylor, J. (1989), 'Preliminary experiments with a distributed multimedia problem solving environment', Proceedings of the First European Conference on Computer-supported Cooperative Work (EC-CSCW '89), London, 13-15 September, 19-34.

Whitelock, D. and Scanlon, E. (1996), 'Motivation, media and motion: reviewing a computer supported collaborative learning experience', in P. Brna, A. Paiva and J. Self (eds.), Proceedings of the European Conference on Artificial Intelligence in Education, Brighton: AIED.

Whitelock, D., del Soldato, T., Scanlon, E. and Taylor J. (1996), 'Moons and collisions: how different tasks influence collaborative problem-solving in science using information technology', paper presented at European Conference on Educational Research, University of Seville, Spain. 